\title{
Subjective ratings of sibling psychological similarity
}

Baskaeva O.V. ${ }^{1}$, Pyankova S.D. ${ }^{2}$

${ }^{1}$ FGBNU "Psychological Institute of Russian Academy of Education", Moscow, Russia

${ }^{2}$ Lomonosov Moscow State University, Moscow, Russia

Mailto: baskaeva.oksana@gmail.com ; pyankova@gmail.com

\section{INTRODUCTION}

Self-perception and perception of the partner in sibling pairs were studied.

A particular way siblings percept psychological peculiarities of each other reflects a level of mutual identification, influences relationships in a pair and their level of social adaptation.

Goal: to estimate a level of perceptible similarity between oneself and one's sibling by personal characteristics.

\section{Method}

\section{PARTICIPANTS}

Sample: 151 pairs siblings; 302

participants; 196 women, 106 men.

Two subgroups: the age of the older siblings was $29.0 \pm 8.52$ years, the age of the younger siblings was $26.1 \pm 8.30$ years.

\section{PROCEDURE}

Participants were offered to fill in four questionnaires according to two instructions.
Instruction 1:

"Estimate a level of agreement with each statement."

Instruction 2 required filling the questionnaire for one's sibling: "How do you think your brother/sister could answer this question?"

Therefore, every participant had two types of indicators:

$>$ self-assessment,

$>$ assessment from the partner.

\section{MEASURES}

$>$ Eysenck Personality Inventory (EPI),

$>$ Locus of Control Questionnaire (LCQ),

D Dark Triad Questionnaire,

$>$ Short Big Five (B5).

\section{DATA ANALYSIS}

The Spearman's rank correlation coefficient of similarly-named indicators in two subgroups were calculated.

\section{RESULTS}

Statistically significant correlations between self-assessments and assessment from the partner in both subgroups were found.

Correlations in the younger subgroup are closer:

$>19$ significant correlations:

$>0.27<r<0.74$;

$>\mathrm{p}<0.01$ for 16 correlations,

$>p \leq 0.03$ in three cases.

Correlations in the elder subgroup:

$>14$ significant correlations;

$>0.26<r<0.64$;

$>\mathrm{p}<0.01$ for 9 correlations

$>p \leq 0.04$ in five cases

Self-assessments of younger siblings are significantly correlated with assessments received from elder siblings by all studied indicators, but Openness to experience (Big Five) and the subscale Internality indicator (Locus of control).
In the elder subgroup no significant correlations were found by a general and four subscale Internality indicators (Locus of control), Social Desirability (EPI) and Agreeableness (Big Five).

Correlation coefficients are statistically significant by all Dark Triad indicators in both subgroups.

\section{CONCLUSIONS}

$>$ Following the results of the research, siblings rate psychological similarity of each other highly enough.

> Siblings understand their siblings' self-perception in a relatively accurate way; negative characteristics of each other are assessed more accurately.

Studied peculiarities of sibling psychological status are an important component of the psychological climate in a family in general. 